\title{
LC-MS analysis to determine the biodistribution of a polymer coated ilomastat ocular implant.
}

Abeer H A Mohamed-Ahmed ${ }^{1 *}$, Alastair Lockwood ${ }^{3}$, Hala Fadda ${ }^{2}$, Shivam Madaan ${ }^{1}$, Peng T Khaw ${ }^{3}$, Steve Brocchini ${ }^{1,3}$, Kersti Karu ${ }^{4}$

${ }^{1}$ UCL School of Pharmacy, 29/39 Brunswick Square, London WC1N 1AX, UK

${ }^{2}$ Department of Pharmaceutical Sciences, College of Pharmacy and Health Sciences, Butler University, Indianapolis, IN 46208, USA

${ }^{3}$ National Institute for Health Research (NIHR) Biomedical Research Centre at Moorfields Eye Hospital NHS Foundation Trust and UCL Institute of Ophthalmology, London EC1V 2PD, UK

${ }^{4}$ UCL Chemistry Mass Spectrometry Facility, Department of Chemistry, Christopher Ignold Building, 20 Gordon Street, London WC1H 0AJ, UK

Corresponding author: Dr. Abeer Mohamed-Ahmed (abeer.ahmed@ucl.ac.uk). 


\begin{abstract}
Ilomastat is a matrix metalloproteinase inhibitor (MMPi) that has shown the potential to inhibit scarring (fibrosis) by mediating healing after injury or surgery. A long lasting ocular implantable pharmaceutical formulation of ilomastat is being developed to mediate the healing process to prevent scarring after glaucoma filtration surgery. The ilomastat implant was coated with water permeable and biocompatible phosphoryl choline polymer (PC1059) displayed extended slow release of ilomastat in vitro and in vivo. The ocular distribution of ilomastat from the implant in rabbits at day 30 post surgery was determined by the extraction of ilomastat and its internal standard marimastat from the ocular tissues, plasma, aqueous humour and vitreous fluid followed by capillary-flow liquid chromatography (cap-LC), the column effluent was directed into a triple quadrupole mass spectrometer operating in product scan mode. The lower limits of quantification (LLOQs) were $0.3 \mathrm{pg} / \mu \mathrm{L}$ for ocular fluids and plasma, and $3 \mathrm{pg} / \mathrm{mg}$ for ocular tissues. The extraction recoveries were 90-95\% for ilomastat and its internal standard from ocular tissues. Ilomastat was found in ocular fluids and tissues at day 30 after surgery. The level of ilomastat was 18 times higher in the aqueous humour than vitreous humour. The concentration ranking of ilomastat in the ocular tissues was sclera $>$ bleb conjunctiva > conjunctiva (rest of the eye) > cornea. Mass spectrometry analysis to confirm the presence of ilomastat in the ocular tissues and fluids at day 30 post-surgery establishes the extended release of ilomastat can be achieved in vivo, which is crucial information for optimisation of the ilomastat coated implant.
\end{abstract}

Keywords: Inhibitors of matrix metalloproteinase, controlled release, ocular biodistribution, mass spectrometry, liquid chromatography. 


\section{Introduction}

Glaucoma is the leading cause of irreversible blindness worldwide [1,2]. The main treatment is to lower the intraocular pressure (IOP), and in many circumstances surgery to create a new drainage channel into the conjunctiva can be effective [3] after the failure of first line treatments involving eye drops. However, scarring in the conjunctiva at the site of surgery blocks aqueous outflow resulting in high IOP and disease progression. Cytotoxic drugs (e.g. mitomycin C) applied locally at the site of surgery can increase success but may have blinding complications, including hypotony and infections [4-6]. Modifications in the technique to administer the cytotoxic drug during surgery can reduce risk, though considerable monitoring is often required postoperatively [3].

Matrix metalloproteinases (MMPs) are zinc-dependent endoproteases that degrade proteins in the extracellular matrix (ECM) playing a crucial role in tissue remodeling during healing in both normal and pathological conditions [7-10]. Inhibitors of matrix metalloproteinases (MMPi) may be a less toxic anti-scarring alternative to mitomycin $\mathrm{C}$ to stop scarring after glaucoma surgery $[2,11]$.

Ilomastat is a synthetic broad spectrum MMPi (Mw $388.5 \mathrm{~g} / \mathrm{mole}, \log \mathrm{P} 0.9$, pKa 8.9) (Figure 1). Preclinical studies by repeated sub-conjunctival injections have shown that ilomastat can inhibit conjunctival scarring after glaucoma surgery in rabbits [2, 11]. Ilomastat can also reduce corneal damage caused by alkali burns in rabbits [12]. Ilomastat has been proven to be safe and effective in Phase I/II trials in patients with bacterial keratitis induced corneal damage using topical ilomastat $[13,14]$. There is currently no ophthalmic pharmaceutical dosage form for ilomastat available in the clinic. To avoid the rapid clearance of injections into the sub-conjunctival space $[15,16]$, we have previously developed an implantable subconjunctival mini implant containing ilomastat for slow, prolonged release at the ocular surgical site [17, 18]. An improved ilomastat implant (1 mg, $1 \mathrm{~mm})$ coated with a water permeable biocompatible phosphoryl choline polymer (PC1059) has been developed to minimize foreign body reactions [19]. Since the implant is designed to slowly release ilomastat over a 3-4 week period, it was necessary to quantify the amount of ilomastat in the ocular fluids and tissues. Knowledge of the ocular distribution of ilomastat from the implant is necessary for continued development. Upon release of the drug, the remaining PC1059 is designed to remain at the site of surgery as a spacer in the conjunctival space to promote aqueous outfl $\boldsymbol{A}$ 

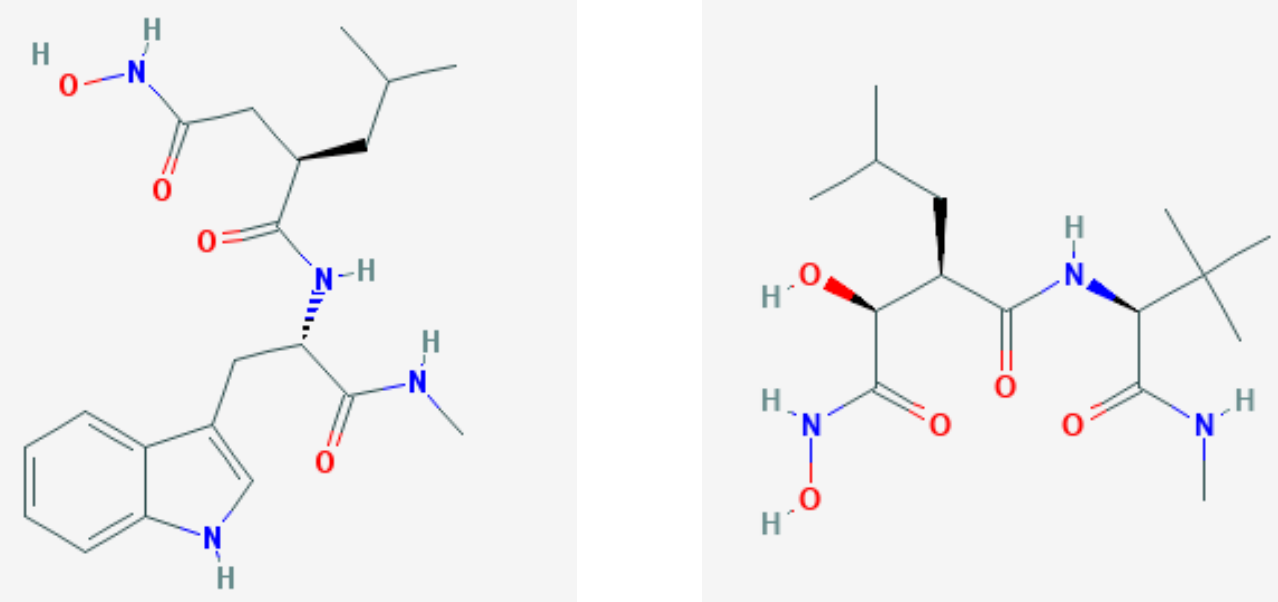

Figure 1. Chemical structure of ilomastat (A) and marimastat (B). https://pubchem.ncbi.nlm. nih.gov/compound.ow.

Currently there is no well-established analytical method to quantify ilomastat in biological tissues and fluids, especially in the eye. Several studies describe analytical techniques such as high performance liquid chromatography (HPLC) and UV-VIS spectroscopy to quantify ilomastat in vitro [18, 20-22]. For example, non-covalently bound ilomastat to streptavidin was quantified using fluorescence spectroscopy in vitro [23]. The in vitro permeation of ilomastat across mice abdominal skin membrane was quantified in vitro by HPLC (detection limit $60 \mathrm{ng} / \mathrm{mL}$ ) [22]. In studies by Magnus et al. (1999), the amount of ilomastat in wound fluids in pigs following topical administration was quantified using HPLC, but details of the HPLC protocol were not described [21]. Other studies have used imaging to visualise the activity of MMPs after administration of ilomastat in vivo [24, 25]. Wang et al. (2009) has used LC/MS (limit of detection of $1 \mu \mathrm{M}$ ) to quantify MMP-1 and ilomastat following micro-dialysis co-perfusion of the enzyme and ilomastat in rats [26].

We have developed a LC-MS method to quantify ilomastat in ocular tissues which offers significant advantages over existing UV based detection techniques in terms of sensitivity and selectivity. To determine the ocular distribution of ilomastat, the aqueous humour, vitreous fluid, ocular tissues and plasma were collected from rabbits. We established and validated an isolation method based on solid phase extraction for plasma samples and liquid-liquid extraction for ocular tissues/fluids samples. A capillary-LC system connected to a TSQ Quantum Access triple quadrupole mass spectrometer operated in the parent scan mode utilising selective reaction monitoring (SRM) set at $\mathrm{MS}^{2}$ transitions for ilomastat and internal standard (marimastat, Mw $331.41 \mathrm{~g} / \mathrm{mole}$, $\log$ P 0.5, Figure 1) was used to quantify ilomastat in the ocular tissues and fluids after an implant period of 30 days in the 
subconjunctival space of the rabbit eye. This cap-LC-MS method gives sub-pico gram (subpg) sensitivity for ilomastat measurement (on-column).

The main objectives of this study were; (1) to develop LC-MS method for analysis of ilomastat in ocular tissues and fluids, (2) to determine the ocular and plasma distribution of ilomastat from the implant in rabbits, and (3) to characterise the ilomastat coated implant in vitro and in vivo to demonstrate that extended ilomastat release can be achieved.

\section{Materials and Methods}

\subsection{Materials}

Ilomastat (98\% purity) and the internal standard marimastat (98\% purity) were purchased from Ryss Lab (Union City, CA, USA). Phosphoryl choline polymer (PC-1059) was donated by Vertellus Biomaterials (Basingstoke, UK). The chemical structure of ilomastat and marimastat are shown in Figure 1. HPLC grade water, methanol and acetonitrile, ethyl acetate and diethyl ether were purchased from Fisher Scientific (Loughborough, UK). Acetic acid (99\% purity) and formic acid were purchased from Sigma Aldrich (Gillingham, UK). Proteinase K was purchased from Promega (Southampton, UK).

\subsection{Methods}

\subsubsection{Preparation and characterization of ocular implant}

The ilomastat implant implants were fabricated by direct compression using ilomastat only (1 $\mathrm{mm}$ punch and die set (UCL, London, UK)). No lubricant was required between pressings. Approximately $1.2 \mathrm{mg}$ of ilomastat powder was weighed, placed into the $1 \mathrm{~mm}$ punch and die, and then compressed at 0.4 bars for 10 seconds. Implants were dipped coated with a water permeable phosphoryl choline polymer (PC1059) [19] by Vertellus Biomaterials (Basingstoke, UK) and were again weighed in individual Eppendorf tubes before sterilization, which in all cases was conducted by exposure to gamma irradiation ( $25 \mathrm{kGy})$. The coating thickness was determined using Scan Electron Microscopy, SEM (Philips CM 120 Bio-Twin, UCL, London, UK).

\subsubsection{Release of ilomastat from ocular implant}

The drug release studies was performed using an in-house open flow cell system $(200 \mu \mathrm{L})$ [18]. The release media was phosphate buffer saline $(\mathrm{pH} 7.4)$ at flow rate of $2 \mu \mathrm{L} / \mathrm{min}$ to simulate typical flow of aqueous humour [27]. The experiment was conducted at $35^{\circ} \mathrm{C}$ to mimic temperature of subconjunctival space [28]. Samples flows out of the cell were collected and analyzed by HPLC. 
The analysis of ilomastat was performed on the Agilent 1200 series HPLC system (Agilent, USA) equipped with an autosampler, a degasser and two SL bin-pumps and chromatographic separation was achieved on a Synergi Polar-RP Phenomenex column $(4 \mu \mathrm{m}$ $15 \mathrm{~cm}$ ) at UCL (London, UK). The mobile phases were (A) $0.1 \%$ trifluoroacetic acid (TFA) in water and (B) acetonitrile. A linear gradient was used that went from $80 \%$ A to $70 \% \mathrm{~B}$ over 17 minutes. The flow rate was $1 \mathrm{~mL} / \mathrm{min}$ and UV detection was set at $280 \mathrm{~nm}$.

\subsubsection{Differential scan calorimetry analysis (DSC)}

The compatibility between ilomastat and the polymer was studied using differential scan calorimetry (DSC-Q2000, UCL, London, UK) and high performance chromatography (HPLC) at UCL (London, UK). The polymer PC1059 film was dipped in ilomastat solution $(100 \mu \mathrm{M})$ in PBS (pH 7.4) for 30 mins, then washed with water and left to dry overnight. The control blank polymer was prepared following similar method using PBS without ilomastat. The polymer films were then scanned at DSC from -100 to $250{ }^{\circ} \mathrm{C}$ at a temperature ramp rate of $3{ }^{\circ} \mathrm{C} / \mathrm{min}$.

\subsubsection{Implantation of ilomastat implant and sample acquisition}

The in vivo ocular distribution of the coated ilomastat mini implant implant $(1 \mathrm{mg}, 1 \mathrm{~mm}$ diameter) was studied in six white female New Zealand rabbits. These experiments were performed in accordance with the ARVO Statement for the Use of Animals in Ophthalmic and Vision Research. Coated ilomastat implant was placed sub-conjunctively in the left rabbit eye. The rabbit was positioned on its right side and povidone-iodine instilled into the left eye. The nictitating membrane was excised. A partial thickness 8-O silk corneal traction suture (Ethicon) was placed superiorly and the eye infraducted. A fornix-based conjunctival flap is then raised and a blunt dissection exposed sclera to approximately $15 \mathrm{~mm}$ behind the limbus. An MVR blade was passed through the sclera at a shallow angle to enter the anterior chamber tangentally to the iris and then withdrawn. A 22 gauge / $25 \mathrm{~mm}$ cannula was then passed along the tract created by the blade and sutured to the scleral surface with a single 10-O nylon suture. The cannula was trimmed and beveled so that it extended $1 \mathrm{~mm}$ above the scleral surface. Ilomastat implant was placed directly behind the tube. The conjunctiva was replaced and closed laterally with two purse string sutures; further povidone-iodine was applied to minimize the risk of infection. Chloramphenicol ointment was instilled at the end of surgery. After 30 days, the rabbits were sacrificed and eyes were removed. Ocular tissues and fluids were collected immediately after removal of rabbit eyes and frozen. Blood samples were collected at day 3, 18 and 30 post-surgery. Plasma was separated immediately by 
centrifugation (10,000 rpm for 10 mins) and was frozen immediately. The frozen tissues and fluids were stored at $-80^{\circ} \mathrm{C}$ until the time of cap-LC-MS analysis. The polymeric spacer implant was removed after 30 days and incubated in methanol to dissolved remaining ilomastat which was then analyzed by HPLC.

\subsubsection{Extraction of ilomastat from rabbit plasma by solid phase extraction (SPE)}

The marimastat internal standard (IS) solution $(10 \mu \mathrm{L})$ in methanol $(1 \mathrm{ng} / \mu \mathrm{L})$ was added to rabbit plasma $(200 \mu \mathrm{L})$ and vortexed $(2 \mathrm{~min})$. Plasma proteins were precipitated by adding $800 \mu \mathrm{L}$ of methanol followed by vortexing $(2 \mathrm{~min})$ and then centrifugation at $6,000 \mathrm{rpm}$ for 20 min (at ambient room temperature). The supernatant was collected and then ilomastat/IS were extracted using solid phase extraction cartridge (Sep-Pak-C18, 1 g, Waters, Elstree. UK) (SPE) as follows; the SPE cartridge was hydrated with methanol $(3 \mathrm{~mL})$ followed by washing with water $(3 \mathrm{~mL})$. Plasma sample (supernatant) was loaded in to the column and washed with water containing $2 \%$ acetic acid ( $3 \mathrm{~mL}$, three times). Ilomastat and IS were found to be retained on the SPE cartridge even after 4 column washes, while salts and more polar metabolites eluted in the flow-through and first column washes. The ilomastat and the IS was then eluted using methanol containing 2\% acetic acid (1 mL) (Fraction 1), followed by methanol (2 mL) (Fraction 2 and 3). The fractions (1-3) were combined and dried by evaporation at $40^{\circ} \mathrm{C}$ under nitrogen. The dried material was then reconstituted in $100 \mu \mathrm{L}$ aqueous acetonitrile (50\%) containing $0.1 \%$ formic acid and then analyzed by cap-LC-MS. For validation of the SPE protocol, blank rabbit plasma $(200 \mu \mathrm{L})$ was spiked with $12.5 \mu \mathrm{L}$ of $0.1 \mathrm{ng} / \mu \mathrm{L}$ ilomastat and IS in methanol and the sample was processed as above.

The calibration curve was prepared using serial concentrations of ilomastat $(0.31,0.63$, $1.5,2.5,5$ and $10 \mathrm{pg} / \mu \mathrm{L})$ each spiked into a blank rabbit plasma (200 $\mu \mathrm{L})$ containing $10 \mu \mathrm{L}$ of $1 \mathrm{ng} / \mu \mathrm{L}$ of the IS. The calibration standard solutions were vortexed and processed as described above.

2.2.6 Extraction of ilomastat from ocular fluids (aqueous and vitreous humour) for cap-LCMS analysis

The IS $(10 \mu \mathrm{L}, 1 \mathrm{ng} / \mu \mathrm{L})$ in methanol was added to $200 \mu \mathrm{L}$ of rabbit aqueous or vitreous humour and then vortexed $(2 \mathrm{~min})$. The proteins were precipitated by the addition of methanol $(400 \mu \mathrm{L})$. The sample was vortexed $(5 \mathrm{~min})$ and then centrifuged at 4,500 rpm at ambient temperature for 20 mins. The supernatant was removed and dried by evaporation at $40^{\circ} \mathrm{C}$ under nitrogen. The dried material was then reconstituted in $100 \mu \mathrm{L}$ of aqueous acetonitrile (50\%) containing $0.1 \%$ formic acid for analysis by cap-LC-MS. 
For constructing calibration curve, serial concentrations of ilomastat $(1.56,3.125$, $6.25,12.5,25$ and $50 \mathrm{pg} / \mu \mathrm{L})$ were prepared in blank aqueous humour $(200 \mu \mathrm{L})$ which contained $10 \mu \mathrm{L}$ of $1 \mathrm{ng} / \mu \mathrm{L}$ of the IS. The same protocol was followed for constructing calibration curve in vitreous humour, serial concentrations of ilomastat $(0.31,0.63,1.5,2.5,5$ and $10 \mathrm{pg} / \mu \mathrm{L})$ were prepared in blank vitreous fluid $(200 \mu \mathrm{L})$ containing $10 \mu \mathrm{L}$ of $1 \mathrm{ng} / \mu \mathrm{L}$ of the IS. Each calibration standard was processed as described above.

\subsubsection{Extraction of ilomastat from ocular tissues for LC-MS analysis}

Ocular tissues (sclera, conjunctiva and cornea) were dissected then frozen immediately on dry ice and stored at $-80^{\circ} \mathrm{C}$. Tissues were lyophilized and the weights of dry tissues were recorded for cornea $20.15 \pm 1.0 \mathrm{mg}$; sclera $107.53 \pm 8.91 \mathrm{mg}$ and conjunctiva $131.45 \pm 50.81$ mg. Phosphate buffer saline $(800 \mu \mathrm{L}, \mathrm{pH} 7.4)$ and $10 \mu \mathrm{L}$ of marimastat ( $1 \mathrm{ng} / \mu \mathrm{L}$ in methanol) were added to each of the ocular tissues incubated for $1 \mathrm{~h}$ at $55^{\circ} \mathrm{C}$. Proteinase $\mathrm{K}(800 \mu \mathrm{L}$ of 1 $\mathrm{mg} / \mathrm{mL}$ in phosphate buffer, $\mathrm{pH} 7.4$ ) was then added to tissue solution and incubated at $55^{\circ} \mathrm{C}$ for a further $4 \mathrm{~h}$ with shaking $250 \mathrm{rpm}$ [29]. The tissue homogenates were vortexed (5 min). Proteins were precipitated by the addition of methanol $(3.2 \mathrm{~mL})$ followed by the vortexing $(5$ min). The supernatant was removed and diethyl ether $(4.8 \mathrm{~mL})$ was added to precipitate fatty acids. The samples were mixed and centrifuged at 4,500 rpm at ambient temperature for 20 mins. The supernatants were then concentrated in vacuum to approximately a $1 \mathrm{~mL}$ volume and then $2 \mathrm{~mL}$ of water was added to the concentrated samples. This was followed by liquidliquid extraction using ethyl acetate $(9 \mathrm{~mL})$, which was added to the sample followed by vigorous mixing for $48 \mathrm{~h}$ in shaker. The ethyl acetate phase was then carefully collected using glass pipette and dried by the evaporation at $40^{\circ} \mathrm{C}$ under nitrogen. The dried material was then reconstituted in $50 \%$ aqueous acetonitrile $(100 \mu \mathrm{L})$ containing $0.1 \%$ formic acid before the LC-MS analysis.

The calibration curves for ilomastat measurement were prepared using blank ocular tissues (100 mg of sclera, $100 \mathrm{mg}$ of conjunctiva and $20 \mathrm{mg}$ of cornea). For cornea calibration curves, the tissue homogenate was spiked with 62.5, 125, 250, 500 and 1000 pg of ilomastat. The calibration curves for conjunctiva/sclera tissue were prepared by spiking the tissue homogenates with 312.5, 625, 1250, 2500, 5000 and $10000 \mathrm{pg}$ of ilomastat. Marimastat (10 $\mu \mathrm{L}, 1 \mathrm{ng} / \mu \mathrm{L}$ in methanol) was also added to each blank ocular tissue homogenate. All prepared calibration standard solutions were processed as described above for the extraction of ilomastat and marimastat from ocular tissues. 


\subsubsection{Capillary LC-MS analysis (cap-LC-MS)}

The cap-LC-MS analysis was conducted using a Thermo Scientific LC system installed with an Agilent Zorbax Eclipse Plus C18, $3.5 \mu \mathrm{m}, 50 \mathrm{~mm}$ x 2.1mm column (Kings College, London, UK). The mobile phase A consisted $0.1 \%$ formic acid in water and the mobile phase B was $0.1 \%$ formic acid in acetonitrile. The gradient was as follows; $95 \%$ of A for 0.5 min, followed by sharp increase to $95 \%$ B until 6 min, and was left at $95 \%$ B for 1 min. At 7.5 min the mobile phase A was $95 \%$ for the next $2.5 \mathrm{~min}$. The total LC run was $10 \mathrm{~min}$. The flow rate was $200 \mu \mathrm{L} / \mathrm{min}$. The injection volume was $1 \mu \mathrm{L}$. The ilomastat and marimastat eluted from the C18 column at $3.10 \mathrm{~min}$ and $2.96 \mathrm{~min}$, respectively. The effluent from the C18 column was directed to the triple quadruple mass spectrometer (Thermo Scientific, TSQ Quantum Access, Kings College, London, UK).

The selected reaction monitoring (SRMs) scans were set-up as determined from an initial mass spectrometry analysis using the $\mathrm{LCQ}^{\mathrm{duo}}$ cylindrical ion trap mass spectrometer (ESI). The product scan mode and the parent scan mode were used. For the parent and product scan modes SRMs were based on the parent ions at $\mathrm{m} / \mathrm{z} 389.1$ and $\mathrm{m} / \mathrm{z} 332.1$ by transmission through the parent Q1 quadrupole mass analyzer of these parent-ions corresponding to ilomastat and marimastat. Whereas, the second Q2 cell collision was set to fragment the parent-ions at $\mathrm{m} / z, 389.1$ and $\mathrm{m} / \mathrm{z} 332.1$ with an isolation width of 1.0. Collision energies were set at $12 \%$ for ilomastat and $10 \%$ for marimastat. In the parent scan mode, the third Q3 quadrupole mass analyzer was set to transmit only the fragment-ions at $\mathrm{m} / \mathrm{z} 227.1$ and $\mathrm{m} / \mathrm{z}, 356.1$ for ilomastat and at $\mathrm{m} / \mathrm{z} 273.1$ and $\mathrm{m} / \mathrm{z} 301.1$ for marimastat, with the isolation width of 1.5. In the product scan mode, the third Q3 quadrupole mass analyzer was set to scan all fragment-ions and generated product mass spectra through the LC chromatographic run.

The reconstructed ion chromatograms were constructed for SRMs transitions corresponding to ilomastat and marimastat. The quantification of ilomastat in the plasma, ocular tissues and fluids was determined by constructing calibration curves of the ratio (Ilomastat/Marimastat) versus ilomastat concentration $(\mathrm{pg} / \mu \mathrm{L})$.

\section{Results and discussion}

\subsection{Preparation and characterization of ocular implant}

Ilomastat was fabricated as a mini-implant ocular implant (1 $\mathrm{mg}, 1 \mathrm{~mm}$ diameter). The implant was coated with a water permeable biocompatible phosphoryl choline polymer (PC1059) [19]. The coating was found to be uniform that was $2.8 \pm 0.3 \mu \mathrm{m}$ thick in the dry 
state (Figure 2). Upon wetting, the polymer coating swells to approximately $5.0 \pm 0.5 \mu \mathrm{m}$ thick.

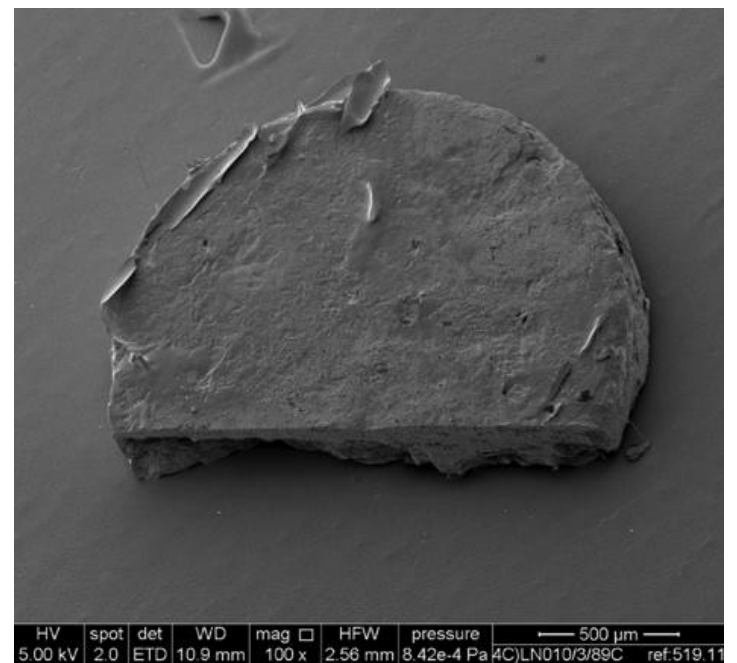

A
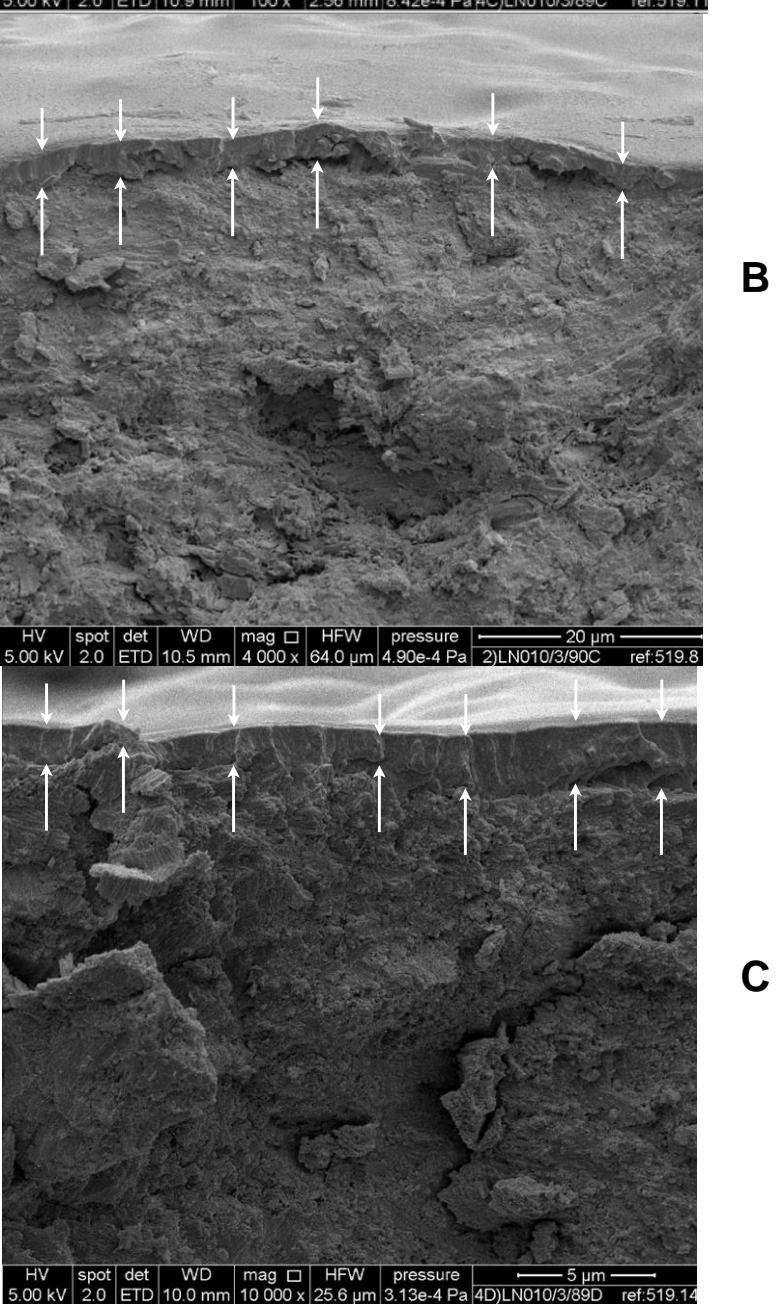

C

Figure 2. Ilomastat implant coated with PC polymer. These SEM images show three different implants coated with PC1059. White arrows are used to show the coating boundaries. (A) Top view of an implant after fracture (scale is $500 \mu \mathrm{m}$ ); (B) and (C) side-on views of two different implants at increasing magnification (scale is 20 and $5 \mu \mathrm{m}$ respectively). The polymer coating for the implant is uniform with thickness of $2.8 \pm 0.3 \mu \mathrm{m}$ thick in the dry state. 
DSC analysis showed no incompatibility between ilomastat and the polymer. The glass transitions of the PC1059 film alone and the polymer film after exposure to ilomastat solution (100 $\mu \mathrm{M}$ in PBS, $30 \mathrm{~min}$ ) were similar (Figure 3A). The absence of incompatibility between the drug and polymer was also confirmed by HPLC, where the retention time of processed ilomastat for both the uncoated and coated implants was $6.6 \mathrm{~min}$, which is the same as that for unprocessed ilomastat prior to implant fabrication and (Figure 3B). Furthermore, no degradation products (additional peaks) were observed by HPLC (Figure $3 \mathrm{C})$.

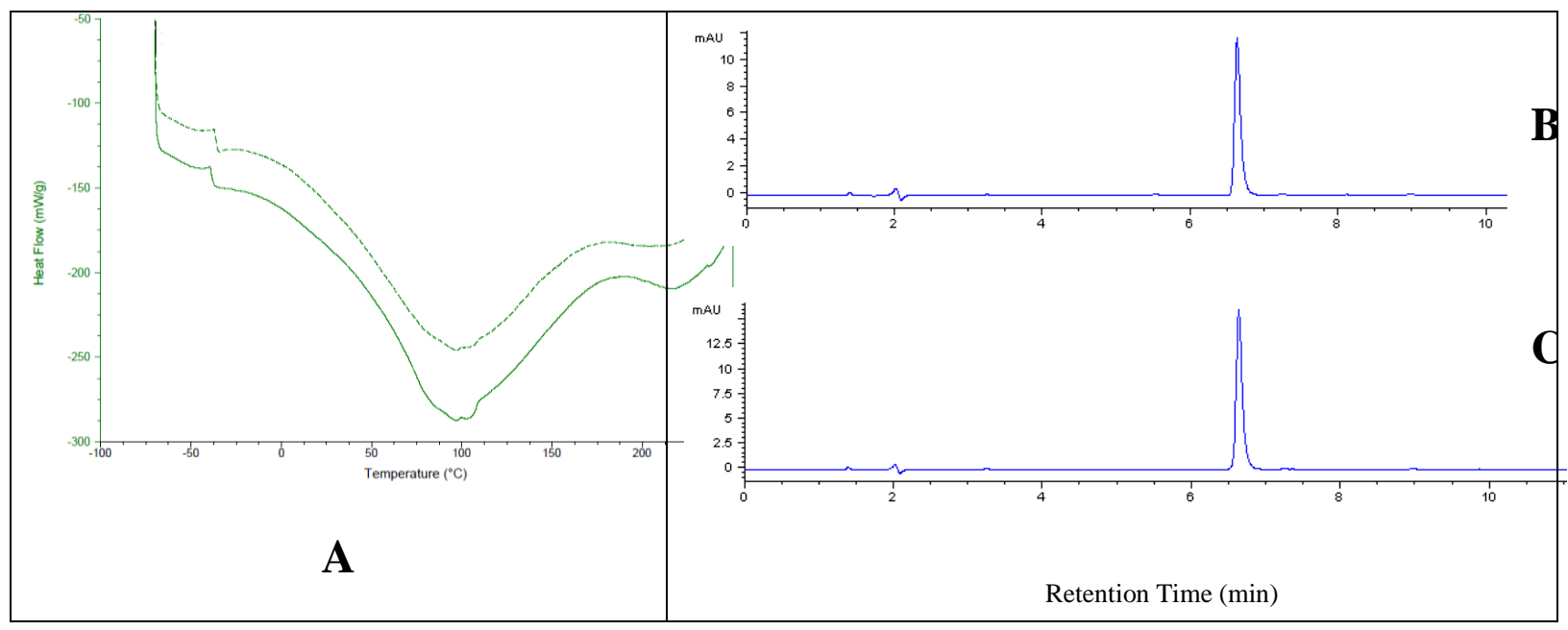

Figure 3. Evaluation of the chemical composition of polymer and ilomastat. (A) DSC trace of PC1059 film +/- exposure to ilomastat solution (100 $\mu \mathrm{M}$ in PBS, $30 \mathrm{~min})$ (dotted line) and blank PC1059 film (solid line) showing similar Tg of the polymer with/out hydration in PBS. HPLC traces are shown for an uncoated (B) and PC-1059 coated (C) ilomastat implant. The retention time for both the uncoated and coated implants is $6.6 \mathrm{~min}$ and there are no degradation peaks observed in the chromatogram after coating the implant.

The release of ilomastat from the coated implant in PBS $(\mathrm{pH} 7.4)$ at $35^{\circ} \mathrm{C}$ was examined using a flow cell designed to simulate the sub-conjunctival aqueous outflow (volume, aqueous flow rate and fluid composition) [18]. Prolonged ilomastat release from the implant within the therapeutic range was observed over 21 days $(21.32 \pm 2.81$ to $39.0 \pm 4.82$ $\mu \mathrm{M}$ ) (Figure 4). The phosphoryl choline polymer coating did not affect the release of ilomastat from the implant and showed a similar release profile as the uncoated ilomastat implant implant [18]. The amount released from the coated ilomastat implant was $66.25 \pm 0.99 \%$ which is similar to uncoated pure ilomastat tablet $(\sim 70 \%)$ after 21 days. This is mainly because the polymer is water permeable and was capable to hydrate quickly after contact with aqueous fluids [19]. 


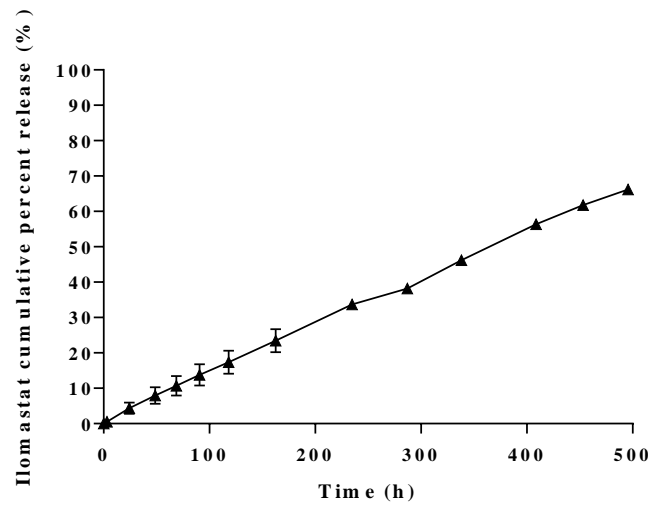

A

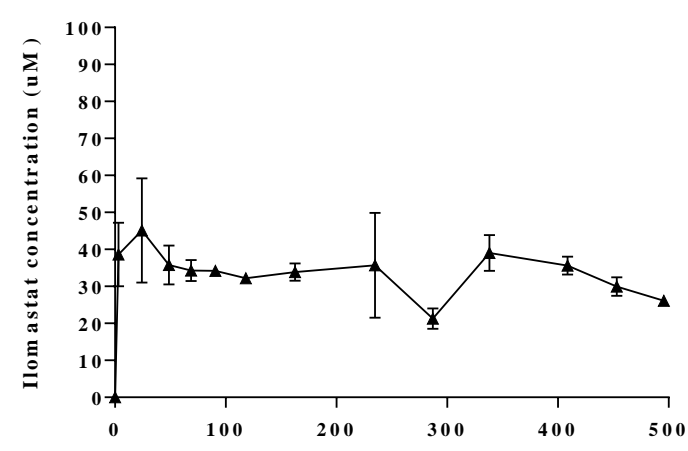

B

Figure 4. Release profiles of PC-coated ilomastat implants in an open flow cell system designed to simulate the sub-conjunctival aqueous outflow $(2 \mu \mathrm{L} / \mathrm{min}$, phosphate buffer saline ( $\mathrm{pH} 7.4)$, at $35^{\circ} \mathrm{C}$ ). (A) The 21-day cumulative release profile for ilomastat implants coated with PC-1059 and (B) the observed concentration of released ilomastat at each time point. Data was expressed average $\pm \operatorname{STD}(n=3)$.

\subsection{Ocular distribution of ilomastat}

The ocular distribution of ilomastat in the rabbits was studied using cap-LC-MS analysis. The cap-LC-MS method was established and validated using the ocular tissues and fluids spiked with ilomastat (ESI). The structurally related MMP inhibitor marimastat was included as an internal standard (IS). The lower limits of quantification (LLOQs) were between $0.3 \mathrm{pg} / \mu \mathrm{L}$ and $3 \mathrm{pg} / \mathrm{mg}$ for ilomastat and marimastat in ocular fluids and tissues. The extraction recoveries were $90-95 \%$ for both analytes. The validation of cap-LC-MS method is described in the ESI. This LC-MS method was able to quantify ilomastat in the ocular fluids and tissues.

The ocular tissues were homogenized enzymatically using proteinase $\mathrm{K}$. This enzyme is a serine protease which cleaves peptide bonds adjacent to the carboxylic group of aliphatic and aromatic amino acids [30]. Proteinase $\mathrm{K}$ was found to digest sclera from the Tree Shrew [29]. In our study, the buffer and temperature used for digestion of the rabbit ocular tissues were modified. Phosphate buffer saline $(\mathrm{pH} 7.4)$ and high temperature $\left(55^{\circ} \mathrm{C}\right)$ were used to produce ocular tissue homogenates. Calibration curves for ilomastat spiked into homogenized tissues (cornea, sclera and conjunctiva), aqueous humour and vitreous fluids are shown in ESI (Figures A1-5). The IS marimastat was used to eliminate the effect of drug loss during the extraction procedure and for the quantification of ilomastat in ocular tissues and fluids. ESI Figures A6-10 show the Reconstructed Ion Chromatograms (RICs) for ilomastat and marimastat measured in the ocular tissues and fluids. 
Ilomastat was detected in all of the examined ocular tissues and fluids indicating there was release of ilomastat from the implant in the eye over 30 days. The level of ilomastat in the aqueous humour $(217.73 \pm 194.92 \mathrm{nM})$ was 17 times higher than in the vitreous humour $(15.47 \pm 12.96 \mathrm{nM})$ (Figure 5).

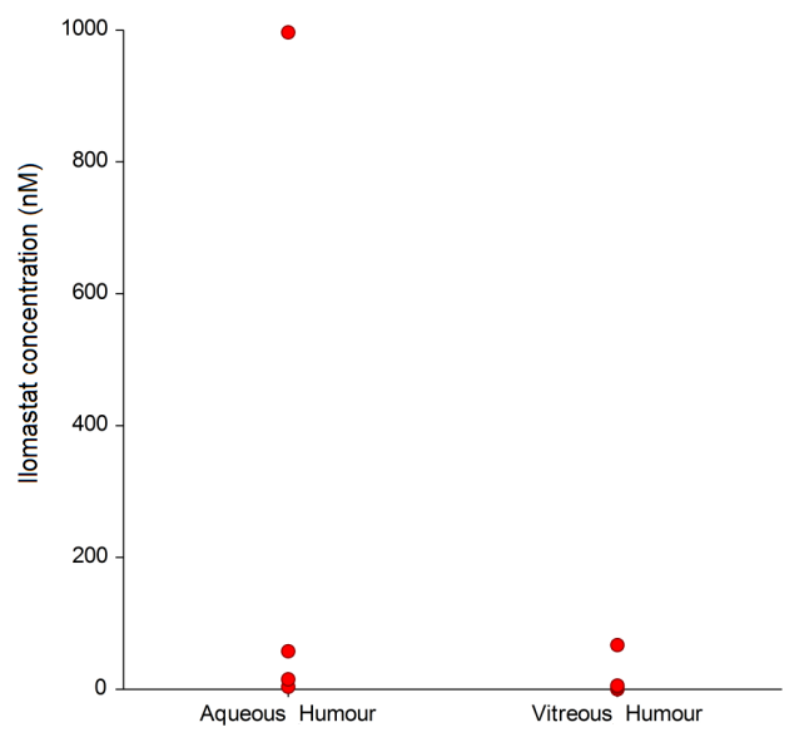

Figure 5. Level of ilomastat in the ocular fluids of the rabbit at day 30 post-surgery. Aqueous humour was removed from the anterior chamber. Ilomastat was extracted from ocular fluids using methanol. Data is presented as a dot plot showing ilomastat levels in each rabbit $(n=5)$.

The amount of ilomastat in the bleb conjunctiva $(719.13 \pm 267.68 \mathrm{pg} / \mathrm{mg}$ tissue) was 8 times higher than the conjunctiva in the rest of the eye $(92.62 \pm 45.83 \mathrm{pg} / \mathrm{mg}$ tissue) (Figure 6). This is desirable as ilomastat is meant to act locally in the conjunctiva tissue surrounding the bleb to prevent scarring at the site of the surgery. The sclera (whole eye) displayed the highest level of ilomastat $(3818.18 \pm 351.17 \mathrm{pg} / \mathrm{mg}$ tissue) compared to other ocular tissues, which might be due to high binding affinity of ilomastat to the sclera. The cornea showed the lowest level of ilomastat $(62.74 \pm 21.92 \mathrm{pg} / \mathrm{mg}$ tissue) (Figure 6). The ilomastat completely dissolved from the implant after 30 days with only a negligible amount of ilomastat remaining $(18.01 \pm 14.26 \mu \mathrm{g} ; 1.8 \pm 1.4 \%$ of the ilomastat from the starting implant) at the site of implantation. The presence of ilomastat at a therapeutic concentration at the site of glaucoma surgery for period up of approximately 4 weeks will avoid the need for multiple sub-conjunctival injections. 


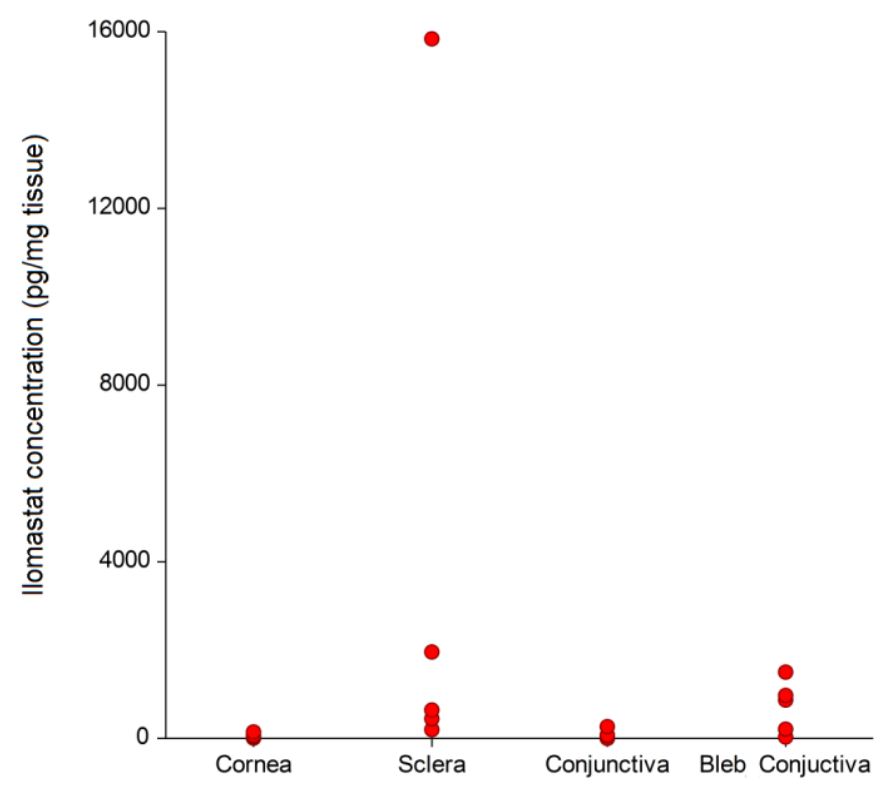

Figure 6. Level of ilomastat in the ocular fluids of the rabbit at day 30 post-surgery. The whole sclera and cornea were removed from the rabbit globe. Bleb conjunctiva was removed and the conjunctiva surrounding the globe was collected separately. Ilomastat was extracted from homogenised ocular tissues. Data is presented as a dot plot showing ilomastat levels in each rabbit $(n=5)$.

The biodistribution study revealed the presence of ilomastat in ocular tissues and fluids. The high level of ilomastat in the aqueous humour is desirable for its local therapeutic effect in the sub-conjunctiva space $[2,11]$. Moreover the concentration of ilomastat in the bleb conjunctiva was higher than in the cornea and the conjunctiva in the rest of the eye. This is also desirable for inducing the local therapeutic effect of ilomastat as the main target tissue is the conjunctiva surrounding the bleb $[2,3,11,31]$. These results confirmed that extended release of ilomastat from the implant can be achieved for an extended period in vivo.

\subsection{Distribution of ilomastat in the rabbit plasma}

The distribution of ilomastat in rabbit plasma was studied using cap-LC-MS analysis. The validation of cap-LC-MS method is described in the ESI section B. Ilomastat and the internal standard (IS, marimastat) were extracted from rabbit plasma samples. Methanol was selected as the preferred solvent for protein precipitation and extraction of ilomastat and IS from a rabbit plasma. The purification of ilomastat from the plasma samples was achieved using a Sep-Pak tC18 (1 g) cartridge. The calibration curve generated by adding known amounts of ilomastat into rabbit plasma is shown in the ESI (Figure A11). The RICs chromatograms for ilomastat and marimastat measured in the rabbit plasma are shown in ESI (Figure A12). The extraction recoveries ranged from $92.5-102.1 \%$ for ilomastat and marimastat. 
The concentration of ilomastat in the rabbit plasma after 30 days post-surgery was negligible $(0.15-0.36 \mathrm{nM})$ as shown in Table 1 . The concentration of ilomastat in the rabbit plasma was found to be similar on day 3 and day 18 post-surgery. The plasma level of ilomastat on day 30 post-surgery was half the concentration as day 3 and day 18 post-surgery. The ilomastat plasma level on day 30 post-surgery was approximately 80 and 1,400 times lower than in the vitreous humour or in the aqueous humour respectively. Ilomastat was primarily localized at the site of implantation with negligible amounts of ilomastat observed in the plasma. The dose used in the ilomastat implant is very small, well below any possible cumulative systemic toxicity that could occur during the release period.

\begin{tabular}{ll}
\hline Post-surgery day & Ilomastat concentration $(\mathbf{n M})$ \\
\hline Day 3 & $0.36 \pm 0.06$ \\
\hline Day 18 & $0.34 \pm 0.21$ \\
\hline Day 30 & $0.15 \pm 0.05$ \\
\hline
\end{tabular}

Table 1. Level of ilomastat in the rabbit plasma (average \pm standard mean error) $(n=5)$.

\section{Conclusions}

A novel, polymer coated ilomastat ocular implant was successfully prepared and displayed extended release of ilomastat in vitro and in vivo. A highly specific and selective cap-LC-MS method have been developed and was able to quantify ilomastat in the ocular fluids and tissues. An in vivo biodistribution study in the rabbit revealed the presence of ilomastat in the ocular fluids and tissues to demonstrate that extended release of ilomastat from the implant in vivo is possible. The level of ilomastat was negligible in the plasma, which indicates that localized ocular delivery of a prolonged therapeutic dose of ilomastat will not be expected to have adverse systemic effects.

\section{Acknowledgement}

We would like to thank Dr. Anna Caldwell and Mr. Tobias Krams (Mass Spectrometry Facility, King's College London) for their help with MS sample analysis. We are grateful to Vertellus Biomaterials UK for a donation of PC1059. We are grateful for funding from the National Institute of Health Research (NIHR) Biomedical Research Centre at Moorfields Eye Hospital NHS Foundation Trust and UCL Institute of Ophthalmology, the Helen Hamlyn Trust (in memory of Paul Hamlyn), the Michael and Ilse Katz Foundation, Medical Research Council, Fight for Sight and Freemasons Grand Charity. The authors do not have any financial or commercial interests in respect to the work described in this manuscript. 


\section{References}

[1] A. Greco, M.I. Rizzo, A. De Virgilio, A. Gallo, M. Fusconi, M. de Vincentiis, Emerging Concepts in Glaucoma and Review of the Literature, Am J Med, (2016).

[2] T.T. Wong, A.L. Mead, P.T. Khaw, Prolonged antiscarring effects of ilomastat and MMC after experimental glaucoma filtration surgery, Invest Ophthalmol Vis Sci, 46 (2005) 2018-2022.

[3] T. Van Bergen, S. Van de Velde, E. Vandewalle, L. Moons, I. Stalmans, Improving patient outcomes following glaucoma surgery: state of the art and future perspectives, Clin Ophthalmol, 8 (2014) 857-867.

[4] R.L. Stamper, M.G. McMenemy, M.F. Lieberman, Hypotonous maculopathy after trabeculectomy with subconjunctival 5-fluorouracil, Am J Ophthalmol, 114 (1992) 544-553.

[5] H.D. Jampel, L.R. Pasquale, C. Dibernardo, Hypotony maculopathy following trabeculectomy with mitomycin C, Arch Ophthalmol, 110 (1992) 1049-1050.

[6] R. Parrish, D. Minckler, "Late endophthalmitis"--filtering surgery time bomb?, Ophthalmology, 103 (1996) 1167-1168.

[7] A. Haseeb, T.M. Haqqi, Immunopathogenesis of osteoarthritis, Clin Immunol, 146 (2013) 185-196.

[8] J. Hu, Q.-X. Van den Steen Pe Fau - Sang, G. Sang Qx Fau - Opdenakker, G. Opdenakker, Matrix metalloproteinase inhibitors as therapy for inflammatory and vascular diseases, Nat.Rev.Drug Discov., 2007 Jun;6 (2007) 480-498.

[9] G.L. De, H.I. Van, I. Dekeyster E Fau - Stalmans, I.F. Stalmans, L. Moons, MMPs in the neuroretina and optic nerve: modulators of glaucoma pathogenesis and repair?, Invest Ophthalmol.Vis.Sci., 2014 Mar 28;55 (2014) 1953-1964.

[10] P.S. Burrage, K.S. Mix, C.E. Brinckerhoff, Matrix metalloproteinases: role in arthritis, Front Biosci, 11 (2006) 529-543.

[11] T.T. Wong, A.L. Mead, P.T. Khaw, Matrix metalloproteinase inhibition modulates postoperative scarring after experimental glaucoma filtration surgery, Invest Ophthalmol Vis Sci, 44 (2003) 1097-1103.

[12] T. Kato, S. Saika, Y. Ohnishi, Effects of the matrix metalloproteinase inhibitor GM6001 on the destruction and alteration of epithelial basement membrane during the healing of post-alkali burn in rabbit cornea, Jpn $\mathrm{J}$ Ophthalmol, 50 (2006) 90-95.

[13] R.E. Galardy, M.E. Cassabonne, C. Giese, J.H. Gilbert, F. Lapierre, H. Lopez, M.E. Schaefer, R. Stack, M. Sullivan, B. Summers, et al., Low molecular weight inhibitors in corneal ulceration, Annals of the New York Academy of Sciences, 732 (1994) 315-323.

[14] B. Fingleton, Matrix metalloproteinases as valid clinical targets, Current pharmaceutical design, 13 (2007) 333-346.

[15] X. Liu, S.K. Li, E.K. Jeong, Ocular pharmacokinetic study of a corticosteroid by 19F MR, Experimental eye research, 91 (2010) 347-352.

[16] S.H. Kim, K.G. Csaky, N.S. Wang, R.J. Lutz, Drug elimination kinetics following subconjunctival injection using dynamic contrast-enhanced magnetic resonance imaging, Pharmaceutical research, 25 (2008) 512-520.

[17] Q. Ru, H.M. Fadda, C. Li, D. Paul, P.T. Khaw, S. Brocchini, M. Zloh, Molecular dynamic simulations of ocular tablet dissolution, J Chem Inf Model, 53 (2013) 3000-3008.

[18] G. Parkinson, S. Gaisford, Q. Ru, A. Lockwood, A. Khalili, R. Sheridan, P.T. Khaw, S. Brocchini, H.M. Fadda, Characterisation of ilomastat for prolonged ocular drug release, AAPS PharmSciTech, 13 (2012) 10631072.

[19] H.H. Goreish, A.L. Lewis, S. Rose, A.W. Lloyd, The effect of phosphorylcholine-coated materials on the inflammatory response and fibrous capsule formation: in vitro and in vivo observations, J Biomed Mater Res A, 68 (2004) 1-9.

[20] R.A. Caldwell, N. Vyavahare, E.M. Langan, 3rd, M. LaBerge, Society of Biomaterials Graduate Degree Candidate Student Research Award. Matrix metalloproteinase inhibitor within an absorbable coating for vascular applications: delivery device characterization and reduction of smooth muscle cell proliferation and migration, J Biomed Mater Res A, 67 (2003) 1-10.

[21] M.S. Agren, Matrix metalloproteinases (MMPs) are required for re-epithelialization of cutaneous wounds, Arch Dermatol Res, 291 (1999) 583-590.

[22] L. Senhao, Q. Dongqin, Preparation and in vitro evaluation of an ilomastat microemulsion gel by a selfmicroemulsifying system, Pharmazie, 67 (2012) 156-160.

[23] E. Bourguet, K. Brazhnik, A. Sukhanova, G. Moroy, S. Brassart-Pasco, A.P. Martin, I. Villena, G. Bellon, J. Sapi, I. Nabiev, Design, Synthesis, and Use of MMP-2 Inhibitor-Conjugated Quantum Dots in Functional Biochemical Assays, Bioconjug Chem, 27 (2016) 1067-1081.

[24] X. Zhang, J. Bresee, G.B. Fields, W.B. Edwards, Near-infrared triple-helical peptide with quenched fluorophores for optical imaging of MMP-2 and MMP-9 proteolytic activity in vivo, Bioorg Med Chem Lett, 24 (2014) 3786-3790. 
[25] C.V. Gringeri, V. Menchise, S. Rizzitelli, E. Cittadino, V. Catanzaro, G. Dati, L. Chaabane, G. Digilio, S. Aime, Novel Gd(III)-based probes for MR molecular imaging of matrix metalloproteinases, Contrast Media Mol Imaging, 7 (2012) 175-184.

[26] Y. Wang, D.V. Zagorevski, M.R. Lennartz, D.J. Loegering, J.A. Stenken, Detection of in vivo matrix metalloproteinase activity using microdialysis sampling and liquid chromatography/mass spectrometry, Anal Chem, 81 (2009) 9961-9971.

[27] J.W. McLaren, Measurement of aqueous humor flow, Experimental eye research, 88 (2009) 641-647.

[28] S. Kawasaki, S. Mizoue, M. Yamaguchi, A. Shiraishi, X. Zheng, Y. Hayashi, Y. Ohashi, Evaluation of filtering bleb function by thermography, The British journal of ophthalmology, 93 (2009) 1331-1336.

[29] N.A. McBrien, P. Lawlor, A. Gentle, Scleral remodeling during the development of and recovery from axial myopia in the tree shrew, Investigative ophthalmology \& visual science, 41 (2000) 3713-3719.

[30] E. Kraus, U. Femfert, Proteinase K from the mold Tritirachium album Limber. Specificity and mode of action, Hoppe Seylers Z Physiol Chem, 357 (1976) 937-947.

[31] J.T. Daniels, A.D. Cambrey, N.L. Occleston, Q. Garrett, R.W. Tarnuzzer, G.S. Schultz, P.T. Khaw, Matrix metalloproteinase inhibition modulates fibroblast-mediated matrix contraction and collagen production in vitro, Invest Ophthalmol Vis Sci, 44 (2003) 1104-1110. 